\title{
Génération d'un continuum de lumière blanche pour l'analyse spectrale résolue en temps de milieux très diffusants
}

\author{
S. Mottin, C. Jonin, E. Baubeau et P. Laporte \\ Laboratoire Traitement du Signal et Instrumentation, UMR 5516 du CNRS, \\ 23 rue du Docteur Michelon, 42023 Saint-Etienne cedex 02, France
}

\begin{abstract}
Résumé : Une nouvelle technique kilohertz de mesure de temps de vol picoseconde sur un large domaine spectral (I80 nm) est détaillée. Le dispositif se compose d'un laser femtoseconde avec amplitication à dérive de fréquence et d'une caneéra a balay age de

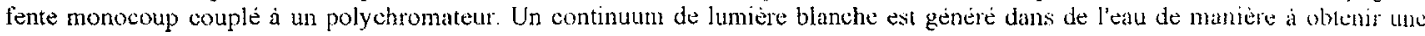
source $450-750 \mathrm{~nm}$ la plus intense. Les images obtenues permettent une analyse spectrale et temporelle simultanée dont la résolution en temps de vol est inférieure a $5 \mathrm{~mm}$ pour un temps d'intégration inférieur à 60 secondes.
\end{abstract}

\section{INTRODUCTION}

Dès 1966, les premières études optiques transcrâniennes chez les enfants hydrocéphales ont été initiées [1]. De nombreux développements de systèmes de neurodiagnostique optique par analyse spectroscopique multidétecteur et multi-émetteur ont été proposés, mais aucun n'a encore été homologué pour des applications cliniques. Pour dépasser les nombreuses limites de ces systèmes à quelques longueurs d'onde discrètes et pour mieux appréhender la complexité de l'optique tissulaire in vivo, nous proposons un dispositif expérimental permettant une étude spectrale ( $350 \mathrm{~nm}-900 \mathrm{~nm}$ par pas de $200 \mathrm{~nm}$ ) résolue en temps (picoseconde). Un autre dispositif expérimental couplant une caméra à balayage de fente et la génération d'un continuum a été décrit dans la littérature [2]. Ce dispositif mettait en œuvre un laser femtoseconde à $10 \mathrm{~Hz}$ de $35 \mathrm{~mJ}$. A cette faible cadence, deux inconvénients majeurs apparaissent pour les applications médicales et biologiques : i) une forte énergie par impulsion, ii) un temps de mesure supérieur à 5 minutes. Le dispositif que nous proposons vise à dépasser ces deux limites.

\section{DISPOSITIF EXPERIMENTAL}

La source du dispositif expérimental est une chaîne laser femtoseconde amplifiée (Alpha 1000-BMI). En sortie du compresseur les impulsions ont les caractéristiques suivantes : 200fs (mode femtoseconde) ou 800fs (mode picoseconde ), 0.5mJ @ $1 \mathrm{KHz}$. Deux longueurs d'onde ont été utilisées : $760 \mathrm{~nm}$ et $800 \mathrm{~nm}$. Le continuum est généré en focalisant le faisceau laser femtoseconde dans une cuve d'eau de $10 \mathrm{~cm}$ de long et de diamètre 1.5 $\mathrm{cm}$. Des focales de 40,100 et $200 \mathrm{~mm}$ ont été utilisées. Un filtre dichroïque réfléchit à plus de $99 \%$ le $800 \mathrm{~nm}$ et transmet la plage spectrale inférieure à $650 \mathrm{~nm}$. Le continuum traverse l'échantillon a étudié. Puis il est transporté par l'intermédiaire d'une fibre de diamètre $0.2 \mathrm{~mm}$. Finalement, il est analysé à travers un spectromètre (270M, Spex Jobin-Yvon) combiné à une streak-caméra permettant la détection du photo-électron unique en 2D. Le domaine spectral est de $182 \mathrm{~nm}$. Cette caméra à balayage de fente (StreakScope C4334, Hamamatsu) est utilisée au temps de déflexion le plus bas égal à 1.127 ns et échantillonnée sur 480 pixels. Le mode d'acquisition est de type monocoup avec un déclenchement à chaque tir laser via une photodiode rapide très sensible (ET2000) permettant d'obtenir plus d'un volt par impulsion afin de diminuer le jitter dû au mode de déclenchement de la caméra.

\subsection{Génération du continuum de lumière blanche}

Le continuum généré est illustré sur la figure 1. Le domaine d'accordabilité est de l'ordre de $500 \mathrm{~nm}$ avec un plateau de 450 à $750 \mathrm{~nm}$. Une chute brutale de signal apparait vers $380 \mathrm{~nm}$ en accord avec la littérature [3] Une puissance moyenne de $120 \mathrm{~mW}$ de continuum a été obtenue sur la fenêtre $350-650 \mathrm{~nm}$ avec la focale de $200 \mathrm{~mm}$. Dans les conditions expérimentales la puissance crête est de l'ordre de $10^{15} \mathrm{~W} / \mathrm{cm}^{2}$. Dans ce cas de nombreux phénomènes non linéaires interviennent et actuellement, il n'y pas de consensus sur la contribution de chacun des phénomènes conduisant à la génération et à l'extinction du continuum. En particulieı l'élargissement spectral reste encore incompris [3]. Dans la présente étude, un des objectifs, a été d'augmenter l'efficacité de production du nombre de photons du proche IR au proche UV. Sur la quantification des rendements des continua la littérature reste discrète. Notre premier choix s'est orienté vers la génération de continuum multifilament dans l'eau. Ce type de source fait encore l'objet d'étude [4-5]. Ce choix a permis d'obtenir une source entre 450 et $650 \mathrm{~nm}$ avec un rendement de $20 \%$ 
Dans nos conditions ce rendement est indépendant du régime ps ou fs. Par contre, les conditions géométriques et la précompression sont très sensibles. L'utilisation d'un milieu liquide permet aussi d'éviter les centres colores et d'autres phénomènes photochimiques. De plus, l'eau présente un gap en énergie de $7.5 \mathrm{eV}$ minimisant l'excitation multiphotonique à $800 \mathrm{~nm}$. Cependant, la qualité spatiale de la source blanche est médiocre (émission conique, multifilamentation...) mais suffisante pour l'application spectroscopique visée. A une puissance moyenne de $0.5 \mathrm{~W}$ en mode ps ou fs, la propagation du train d'impulsions génère des bulles. Elles peuvent être évitées en translatant latéralement la cuve d'eau [4]

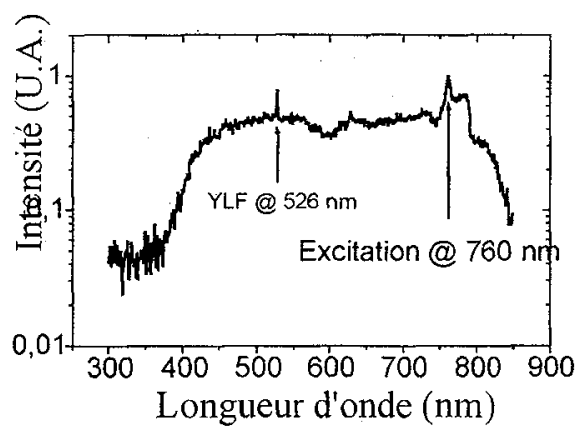

Figure 1: Spectre du continuun? généré dans l'eau Le pic à 526 nm contespond à un parasite dù à la pompe de l'amplificateur.

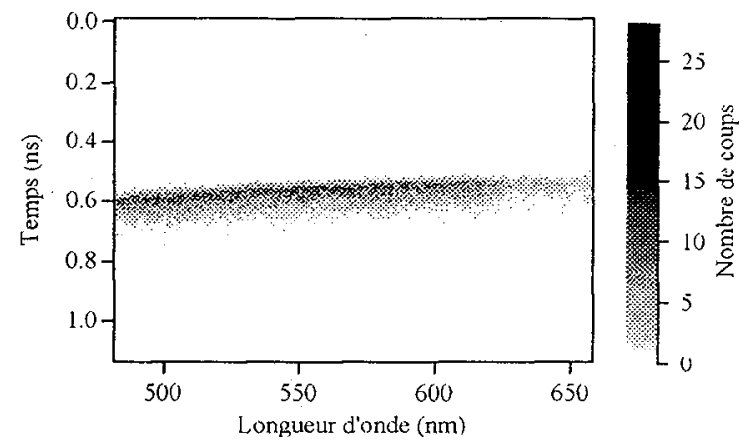

Figure 2 : Image spectrotempolelle du continuum génèrè dans l'eau présentant la fonction appareil du dispositif accumute sut $60 \mathrm{~s}$

L'échelle en gris représente le numbre de pholo-électrons par pixd

La figure 2 illustre la fonction d'appareil du montage expérimental en particulier sa résolution temporelle. Le temps d'intégration de la caméra est fixé à 60 secondes soit 60000 tirs laser induisant un jitter de largeur à mihauteur inférieure à 55 ps alors que la résolution ultime de la streak-camera est inférieure à 10 ps. Dans ce mode accumulatif la limite de l'analyse du temps de vol dans un milieu diffusant aqueux est donc de 5 millimètres de trajet optique après déconvolution. En mode image vidéo $(33 \mathrm{~ms}), 3$ millimètres de résolution peuvent être atteints. Par contre, la limite devient alors le nombre de photo-électrons comptés. Dans un large domaine spectral, le dispositif est conçu pour obtenir simultanément les coefficients d'absorption et de diffusion autorisant la décorrélation des deux phénomènes dans des milieux homogènes. Les mesures sur $3 \mathrm{~cm}^{3}$ de modèles optiques simulant les propriétés optiques cérébrales (non présentées ici) soulignent l'importance d'augmenter le nombre de photons générés par continuum pour les applications chez l'homme où le volume diffusant est 400 fois plus important.

\section{CONCLUSION et PERSPECTIVES}

Nous avons atteint de manière originale une résolution millimétrique avec des temps d'intégration inférieure à 1 minute. La résolution temporelle en mode accumulatif sur une minute de ce dispositif s'avère largement suffisante. Pour le cas des milieux très hétérogènes et en vue de repérer des singularités de la population des diffuseurs ou des absorbeurs, des techniques d'imagerie sont nécessaires et peuvent être obtenues avec le même dispositif par déplacement de la source ou de la fibre optique de collection.

La génération de continuum et l'optimisation des différents modes de confinement spatiaux des lasers ultrabrefs restent l'objet de recherche [6-7]. Notre objectif est d'optimiser le rendement du continuum avec d'autres matériaux et d'autres modes de confinement pour arriver à plus de $400 \mathrm{~mW}$ dans le visible et proche InfraRouge.

\section{Références}

1. D. B. Shurtleff, E. L. Foltz, et D. Fry, Arch. Dis. Childh., 41, 183(1966)

2. S. Andersson-Engels, R. Berg, A. Persson, et S. Svanberg, Opt. Lett., 18, 1697 (1993)

3. A. Brodeur et S. L. Chin, Phys. Rev. Lett, 80, 4406 (1998)

4 A. Brodeur, F. A. Ilkov, et S. L. Chin, Opt Comm., 129, 193 (1996)

5 A. Brodeur et S. L. Chin, J. Opt. Soc. Am. B., 16, 637 (1999)

6 E. E. Fill, J. Opt. Soc. Am. B., 11, 224l (1994)

7 T. A. Birks, W. J. Wadsworth et P. St-J. Russell, CPD 30-1/59,CLEO'2000 